\begin{tabular}{|c|l|}
\hline Title & Evolutes of fronts in the Euclidean plane \\
\hline Author(s) & Fukunaga, Tomonori; Takahashi, Masatomo \\
\hline Citation & Hokkaido University Preprint Series in Mathematics, 1026, 1-17 \\
\hline Issue Date & 2012-12-17 \\
\hline DOI & 10.14943/84172 \\
\hline Doc URL & http://hdl.handle.net/2115/69831 \\
\hline Type & bulletin (article) \\
\hline File Information & pre1026.pdf \\
\hline
\end{tabular}

Instructions for use 


\title{
Evolutes of fronts in the Euclidean plane
}

\author{
T. Fukunaga and M. Takahashi
}

Dedicated to Professor Shyuichi Izumiya on the occasion of his 60th birthday

December 17, 2012

\begin{abstract}
The evolute of a regular curve in the Euclidean plane is given by not only the caustics of the regular curve, envelope of normal lines of the regular curve, but also the locus of singular loci of parallel curves. In general, the evolute of a regular curve have singularities, since such a point is corresponding to a vertex of the regular curve and there are at least four vertices for simple closed curves. If we repeated an evolute, we cannot define the evolute at a singular point. In this paper, we define an evolute of a front and give properties of such evolute by using a moving frame of a front and the curvature of the Legendre immersion. As applications, repeated evolutes can be well-defined and these are useful to recognize the shape of curves.
\end{abstract}

\section{Introduction}

The evolute of a regular plane curve is classical object (cf. $[5,8,9]$ ). It is useful to recognize the vertex of a regular plane curve as a singularity (generically, a $3 / 2$ cusp singularity) of the evolute. The caustics (evolutes) are related as general relativity theory, see for instance [6, 10]. The properties of evolutes discussed by using distance squared functions and the theories of Lagrangian, Legendrian singularity (cf. [1, 2, 3, 13, 14, 17, 20]). Moreover, the singular loci of parallel curves of the regular curve swept out the evolute. By using this property, we can define an evolute of a front in $\S 2$. In order to consider properties of an evolute of a front, we introduce a moving frame of a front (a Legendre immersion) (cf. [7]). In [7], we give an existence and uniqueness for a Legendre curve in the unit tangent bundle like as regular plane curves. It is quite useful to analyse a Legendre curve (or, a frontal) in the unit tangent bundle. In $\S 3$, we give another representation for the evolute of a front by using the moving frame and the curvature of the Legendre immersion (Theorem 3.3). By the representation, we give properties for an evolute of a front, for example, the evolute of a front is also a front. It follows that we can consider the repeated evolute, namely, the evolute of an evolute of a front, see Theorem 4.1 in $\S 4$. Moreover, we extend the notion of the vertex for a front (or, a Legendre immersion) and give a kind of four vertices theorem for a front, see Proposition 3.11. Furthermore, the

2010 Mathematics Subject classification: 58K05, 53A04, 57R45

Key Words and Phrases. evolute, parallel curve, front, Legendre immersion 
evolute of a front is also given by envelope of normal lines of the front. The singular point of the evolute of an evolute of a regular curve measure to the contact of an involute of a circle. In the appendix, we give the condition of contact between regular curves. We give the $n$-th evolute of a front in $\S 5$. In $\S 6$, we give examples of the evolutes of fronts.

All maps and manifolds considered here are differential of class $C^{\infty}$.

Acknowledgement. The second author was supported by a Grant-in-Aid for Young Scientists (B) No. 23740041.

\section{Definitions and basic concepts}

Let $I$ be an interval or $\mathbb{R}$. Suppose that $\gamma: I \rightarrow \mathbb{R}^{2}$ is a regular plane curve, that is, $\dot{\gamma}(t) \neq 0$ for any $t \in I$. If $s$ is the arc-length parameter of $\gamma$, we denote $\boldsymbol{t}(s)$ by the unite tangent vector $\boldsymbol{t}(s)=\gamma^{\prime}(s)=d \gamma / d s(s)$ and $\boldsymbol{n}(s)$ by the unite normal vector $\boldsymbol{n}(s)=J(\boldsymbol{t}(s))$ of $\gamma(s)$, where $J$ is the anticlockwise rotation of $\pi / 2$. Then we have the Frenet formula as follows:

$$
\left(\begin{array}{c}
\boldsymbol{t}^{\prime}(s) \\
\boldsymbol{n}^{\prime}(s)
\end{array}\right)=\left(\begin{array}{cc}
0 & \kappa(s) \\
-\kappa(s) & 0
\end{array}\right)\left(\begin{array}{c}
\boldsymbol{t}(s) \\
\boldsymbol{n}(s)
\end{array}\right)
$$

where $\kappa(s)=\boldsymbol{t}^{\prime}(s) \cdot \boldsymbol{n}(s)$ is the curvature of $\gamma$ and $\cdot$ is the inner product on $\mathbb{R}^{2}$.

Even if $t$ is not the arc-length parameter, we have the unite tangent vector $\boldsymbol{t}(t)=\dot{\gamma}(t) /|\dot{\gamma}(t)|$, the unite normal vector $\boldsymbol{n}(t)=J(\boldsymbol{t}(t))$ and the Frenet formula

$$
\left(\begin{array}{c}
\dot{\boldsymbol{t}}(t) \\
\dot{\boldsymbol{n}}(t)
\end{array}\right)=\left(\begin{array}{cc}
0 & |\dot{\gamma}(t)| \kappa(t) \\
-|\dot{\gamma}(t)| \kappa(t) & 0
\end{array}\right)\left(\begin{array}{c}
\boldsymbol{t}(t) \\
\boldsymbol{n}(t)
\end{array}\right)
$$

where $\dot{\gamma}(t)=d \gamma / d t(t),|\dot{\gamma}(t)|=\sqrt{\dot{\gamma}(t) \cdot \dot{\gamma}(t)}$ and $\kappa(t)=\operatorname{det}(\dot{\gamma}(t), \ddot{\gamma}(t)) /|\dot{\gamma}(t)|^{3}=\dot{\boldsymbol{t}}(t) \cdot \boldsymbol{n}(t) /|\dot{\gamma}(t)|$. Note that $\kappa(t)$ is independent on the choice of a parametrisation.

In this paper, we consider evolutes of plane curves. The evolute $E v(\gamma): I \rightarrow \mathbb{R}^{2}$ of a regular plane curve $\gamma$ is given by

$$
E v(\gamma)(t)=\gamma(t)+\frac{1}{\kappa(t)} \boldsymbol{n}(t)
$$

away from the point $\kappa(t)=0$ (cf. $[5,8,9])$.

If $\gamma$ is not a regular curve, then we can not define the evolute as above, since the curvature may be divergence at a singular point. However, we define an evolute of a front in the Euclidean plane, see Definition 2.9 and Theorem 3.3. It is a generalisation of the evolute of regular plane curves.

We say that $\gamma: I \rightarrow \mathbb{R}^{2}$ is a front (or, a wave front) in the Euclidean plane, if there exists a smooth map $\nu: I \rightarrow S^{1}$ such that the pair $(\gamma, \nu): I \rightarrow \mathbb{R}^{2} \times S^{1}$ is a Legendre immersion, namely, $(\dot{\gamma}(t), \dot{\nu}(t)) \neq(0,0)$ and $(\gamma(t), \nu(t))^{*} \theta=0$ for each $t \in I$. Here $\theta$ is a canonical contact structure on $T_{1} \mathbb{R}^{2}=\mathbb{R}^{2} \times S^{1}$. Remark that the second condition is equivalent to $\dot{\gamma}(t) \cdot \nu(t)=0$ for each $t \in I$ (cf. $[1,2,3])$.

Throughout the paper, we assume that the pair $(\gamma, \nu)$ is co-orientable, the singular points of $\gamma$ are finite and $\gamma$ has no inflection points. First and second conditions can be removed see Remarks 3.4 and 3.5, however, we add these conditions for the sake of simplicity.

We give examples of fronts, other examples see in $[1,4,11]$ etc. 
Example 2.1 One of the typical example of a front is a regular plane curve. Let $\gamma: I \rightarrow \mathbb{R}^{2}$ be a regular plane curve. In this case, we may take $\nu: I \rightarrow S^{1}$ by $\nu(t)=\boldsymbol{n}(t)$. Then it is easy to check that $(\gamma, \nu)$ is a Legendre immersion.

Example 2.2 Let $\gamma: \mathbb{R} \rightarrow \mathbb{R}^{2}$ be a $3 / 2$ cusp $\left(A_{2}\right.$-singularity) given by $\gamma(t)=\left(t^{2}, t^{3}\right)$. In this case, 0 is a singular point of $\gamma$. If we take $\nu: \mathbb{R} \rightarrow S^{1}$ by $\nu(t)=\left(1 / \sqrt{9 t^{2}+4}\right)(-3 t, 2)$, then we can show that $(\gamma, \nu)$ is a Legendre immersion. Hence the $3 / 2$ cusp is an example of a front. The $3 / 2$ cusp is the generic singularity of fronts and also evolutes in the Euclidean plane.

Example 2.3 Let $\gamma: \mathbb{R} \rightarrow \mathbb{R}^{2}$ be a $4 / 3$ cusp ( $E_{6}$-singularity) given by $\gamma(t)=\left(t^{3}, t^{4}\right)$. In this case, 0 is also a singular point of $\gamma$. If we take $\nu: \mathbb{R} \rightarrow S^{1}$ by $\nu(t)=\left(1 / \sqrt{16 t^{2}+9}\right)(-4 t, 3)$, then we can show that $(\gamma, \nu)$ is a Legendre immersion. Hence the $4 / 3$ cusp is also an example of a front, see Example 6.3.

Example 2.4 Let $\gamma: \mathbb{R} \rightarrow \mathbb{R}^{2}$ be a $5 / 2$ cusp $\left(A_{4}\right.$-singularity) given by $\gamma(t)=\left(t^{2}, t^{5}\right)$. In this case, 0 is also a singular point of $\gamma$. However, the $5 / 2$ cusp is not a front. By the condition $\dot{\gamma}(t) \cdot \nu(t)=0$, we take $\nu: \mathbb{R} \rightarrow S^{1}$ by $\nu(t)= \pm\left(1 / \sqrt{25 t^{6}+4}\right)\left(-5 t^{3}, 2\right)$. Then $(\gamma, \nu)$ is not an immersion at $t=0$ and hence $\gamma$ is not a front (but $\gamma$ is a frontal, see [7]).

Remark 2.5 By the definition of the Legendre immersion, if $(\gamma, \nu)$ is a Legendre immersion, then $(\gamma,-\nu)$ is also.

Let $(\gamma, \nu): I \rightarrow \mathbb{R}^{2} \times S^{1}$ be a Legendre immersion. We define a parallel curve $\gamma_{\lambda}: I \rightarrow \mathbb{R}^{2}$ of $\gamma$ by $\gamma_{\lambda}(t)=\gamma(t)+\lambda \nu(t)$ for each $\lambda \in \mathbb{R}$. Then we have following results.

Proposition 2.6 For a Legendre immersion $(\gamma, \nu): I \rightarrow \mathbb{R}^{2} \times S^{1}$, the parallel curve $\gamma_{\lambda}: I \rightarrow \mathbb{R}^{2}$ is a front for each $\lambda \in \mathbb{R}$.

Proof. We take $\nu_{\lambda}: I \rightarrow S^{1}$ by $\nu_{\lambda}(t)=\nu(t)$. Since $\gamma_{\lambda}(t)=\gamma(t)+\lambda \nu(t), \dot{\gamma}_{\lambda}(t)=\dot{\gamma}(t)+\lambda \dot{\nu}(t)$. If $\dot{\gamma}_{\lambda}\left(t_{0}\right)=0$ at a point $t_{0} \in I$, then we have $\dot{\gamma}\left(t_{0}\right)+\lambda \dot{\nu}\left(t_{0}\right)=0$. If $\dot{\nu}_{\lambda}\left(t_{0}\right)=\dot{\nu}\left(t_{0}\right)=0$, then $\dot{\gamma}\left(t_{0}\right)=0$. It is contradict from the fact that $(\gamma, \nu)$ is an immersion and hence $\left(\gamma_{\lambda}, \nu_{\lambda}\right)$ is an immersion. By $\nu(t) \cdot \nu(t)=1$, we have $\dot{\nu}(t) \cdot \nu(t)=0$. Then

$$
\dot{\gamma}_{\lambda}(t) \cdot \nu_{\lambda}(t)=(\dot{\gamma}(t)+\lambda \dot{\nu}(t)) \cdot \nu(t)=\dot{\gamma}(t) \cdot \nu(t)+\lambda \dot{\nu}(t) \cdot \nu(t)=0 .
$$

It follows that $\left(\gamma_{\lambda}, \nu_{\lambda}\right)$ is a Legendre immersion and hence $\gamma_{\lambda}$ is a front.

We denote the curvature of the parallel curve $\gamma_{\lambda}(t)$ by $\kappa_{\lambda}(t)$, when $\gamma_{\lambda}$ is a regular curve.

Proposition 2.7 Let $(\gamma, \nu)$ be a Legendre immersion. If $\gamma$ is a regular curve and $\lambda \neq 1 / \kappa(t)$, then a parallel curve $\gamma_{\lambda}$ is also a regular and $E v\left(\gamma_{\lambda}\right)(t)$ is consistent with $E v(\gamma)(t)$.

Proof. Since $\gamma_{\lambda}(t)=\gamma(t)+\lambda \boldsymbol{n}(t), \dot{\gamma}_{\lambda}(t)=|\dot{\gamma}(t)|(1-\lambda \kappa(t)) \boldsymbol{t}(t)$. By the assumption $\lambda \neq 1 / \kappa(t)$, $\gamma_{\lambda}$ is a regular curve. By a direct calculation, we have

$$
\kappa_{\lambda}(t)=\frac{\kappa(t)}{|1-\lambda \kappa(t)|}, \quad \boldsymbol{n}_{\lambda}(t)=\frac{1-\lambda \kappa(t)}{|1-\lambda \kappa(t)|} \boldsymbol{n}(t) .
$$

Hence

$$
\begin{aligned}
E v\left(\gamma_{\lambda}\right)(t) & =\gamma_{\lambda}(t)+\frac{1}{\kappa_{\lambda}(t)} \boldsymbol{n}_{\lambda}(t)=\gamma(t)+\lambda \boldsymbol{n}(t)+\frac{|1-\lambda \kappa(t)|}{\kappa(t)} \frac{1-\lambda \kappa(t)}{|1-\lambda \kappa(t)|} \boldsymbol{n}(t) \\
& =\gamma(t)+\frac{1}{\kappa(t)} \boldsymbol{n}(t)=E v(\gamma)(t)
\end{aligned}
$$


Remark 2.8 Let $(\gamma, \nu)$ be a Legendre immersion. If $t_{0}$ is a singular point of the front $\gamma$, then $\lim _{t \rightarrow t_{0}}|\kappa(t)|=\infty$. By the equality $\kappa_{\lambda}(t)=\kappa(t) /|1-\lambda \kappa(t)|$, we have $\lim _{t \rightarrow t_{0}} \kappa_{\lambda}(t) \neq 0$, see also Remark 3.2.

We now define an evolute of a front in the Euclidean plane.

Definition 2.9 Let $(\gamma, \nu): I \rightarrow \mathbb{R}^{2} \times S^{1}$ be a Legendre immersion. We define an evolute $\mathcal{E} v(\gamma): I \rightarrow \mathbb{R}^{2}$ of the front $\gamma$ as follows:

$$
\mathcal{E} v(\gamma)(t)= \begin{cases}\gamma(t)+\frac{1}{\kappa(t)} \boldsymbol{n}(t) & \text { if } t \text { is a regular point, } \\ \gamma_{\lambda}(t)+\frac{1}{\kappa_{\lambda}(t)} \boldsymbol{n}_{\lambda}(t) & \text { if } t \in\left(t_{0}-\delta, t_{0}+\delta\right), t_{0} \text { is a singular point of } \gamma,\end{cases}
$$

where $\delta$ is a sufficiently small positive real number and $\lambda \in \mathbb{R}$ is satisfied the condition $\lambda \neq$ $1 / \kappa(t)$.

Remark 2.10 By the assumption of the finiteness of singularities of a front, there exists $\lambda \in \mathbb{R}$ with the condition $\lambda \neq 1 / \kappa(t)$. Moreover, by Proposition 2.7, we can glue on the regular interval of $\gamma$ and $\gamma_{\lambda}$. Then the evolute of a front is well-defined. Furthermore, by definition, the evolute of a front $\mathcal{E} v(\gamma)$ is a $C^{\infty}$ mapping.

In order to consider properties of the evolute of a front, we need a moving frame of a front (or, a Legendre immersion) (cf. [7]). Let $(\gamma, \nu): I \rightarrow \mathbb{R}^{2} \times S^{1}$ be a Legendre immersion. If $\gamma$ is a regular curve around a point $t_{0}$, then we have the Frenet formula of $\gamma$ in $\S 2$. On the other hand, if $\gamma$ is singular at a point $t_{0}$, then we don't define such a frame. However, $\nu$ is always defined even if $t$ is a singular point of $\gamma$. Therefore, we have the Frenet formula of a front as follows. We put on $\boldsymbol{\mu}(t)=J(\nu(t))$. We call the pair $\{\nu(t), \boldsymbol{\mu}(t)\}$ is a moving frame of a front $\gamma(t)$ in $\mathbb{R}^{2}$ and we have the Frenet formula of a front which is given by

$$
\left(\begin{array}{c}
\dot{\nu}(t) \\
\dot{\boldsymbol{\mu}}(t)
\end{array}\right)=\left(\begin{array}{cc}
0 & \ell(t) \\
-\ell(t) & 0
\end{array}\right)\left(\begin{array}{c}
\nu(t) \\
\boldsymbol{\mu}(t)
\end{array}\right)
$$

where $\ell(t)=\dot{\nu}(t) \cdot \boldsymbol{\mu}(t)$. Moreover, if $\dot{\gamma}(t)=\alpha(t) \nu(t)+\beta(t) \boldsymbol{\mu}(t)$ for some smooth functions $\alpha(t), \beta(t)$, then $\alpha(t)=0$ follows from the condition $\dot{\gamma}(t) \cdot \nu(t)=0$. Hence, there exists a smooth function $\beta(t)$ such that

$$
\dot{\gamma}(t)=\beta(t) \boldsymbol{\mu}(t)
$$

Since $(\gamma, \nu)$ is an immersion, we have $(\ell(t), \beta(t)) \neq(0,0)$ for each $t \in I$. The pair $(\ell, \beta)$ is an important invariant of Legendre curves (or, frontals) in the unit tangent bundle like as the curvature of a regular plane curve, for more detail see in [7]. We call the pair $(\ell, \beta)$ the curvature of the Legendre curve in [7]. In this paper, we always assume that $(\gamma, \nu)$ is a Legendre immersion, so that we call $(\ell, \beta)$ the curvature of the Legendre immersion. The related properties see in $[15,16]$. 


\section{Properties of the evolute of fronts}

In this section, we consider properties of the evolute of fronts. Let $(\gamma, \nu): I \rightarrow \mathbb{R}^{2} \times S^{1}$ be a Legendre immersion with the curvature of the Legendre immersion $(\ell, \beta)$.

First we give a relationship between the curvature of the Legendre immersion $(\ell(t), \beta(t))$ and the curvature $\kappa(t)$ if $\gamma$ is a regular curve.

Lemma 3.1 (1) If $\gamma$ is a regular curve, then $\ell(t)=|\beta(t)| \kappa(t)$.

(2) If $\gamma_{\lambda}$ is a regular curve, then $\ell(t)=|\beta(t)+\lambda \ell(t)| \kappa_{\lambda}(t)$.

Proof. (1) By a direct calculation, $\dot{\gamma}(t)=\beta(t) \boldsymbol{\mu}(t), \ddot{\gamma}(t)=\dot{\beta}(t) \boldsymbol{\mu}(t)-\beta(t) \ell(t) \nu(t)$ and

$$
\kappa(t)=\frac{\operatorname{det}(\dot{\gamma}(t), \ddot{\gamma}(t))}{|\dot{\gamma}(t)|^{3}}=\frac{\operatorname{det}(\beta(t) \boldsymbol{\mu}(t), \dot{\beta}(t) \boldsymbol{\mu}(t)-\beta(t) \ell(t) \nu(t))}{|\beta(t)|^{3}}=\frac{\beta(t)^{2} \ell(t)}{|\beta(t)|^{3}}=\frac{\ell(t)}{|\beta(t)|} .
$$

Therefore we have $\ell(t)=|\beta(t)| \kappa(t)$.

(2) We can also prove by the same calculations of (1).

Remark 3.2 Since $(\ell(t), \beta(t)) \neq(0,0)$, if $t_{0}$ is a singular point of $\gamma$, then $\gamma_{\lambda}$ is a regular curve. By Lemma $3.1(2), \ell\left(t_{0}\right)=\left|\lambda \ell\left(t_{0}\right)\right| \kappa_{\lambda}\left(t_{0}\right)$. It follows from $\lambda \ell\left(t_{0}\right) \neq 0$ that $\kappa_{\lambda}\left(t_{0}\right) \neq 0$.

We give another representation of the evolute of a front by using the moving frame of a front $\{\nu(t), \boldsymbol{\mu}(t)\}$ and the curvature of the Legendre immersion $(\ell(t), \beta(t))$.

Theorem 3.3 Under the above notations, the evolute of a front $\mathcal{E} v(\gamma)(t)$ is represented by

$$
\mathcal{E} v(\gamma)(t)=\gamma(t)-\frac{\beta(t)}{\ell(t)} \nu(t)
$$

and $\mathcal{E} v(\gamma)$ is a front.

Proof. First suppose that $\gamma$ is a regular curve. Since $\dot{\gamma}(t)=\beta(t) \boldsymbol{\mu}(t)$, we have $|\beta(t)| \neq 0$ and

$$
\boldsymbol{t}(t)=\frac{\beta(t)}{|\beta(t)|} \boldsymbol{\mu}(t), \quad \boldsymbol{n}(t)=-\frac{\beta(t)}{|\beta(t)|} \nu(t) .
$$

By Lemma $3.1(1), \kappa(t)=\ell(t) /|\beta(t)|$ and $\ell(t) \neq 0$. Then

$$
\mathcal{E} v(\gamma)(t)=\gamma(t)+\frac{1}{\kappa(t)} \boldsymbol{n}(t)=\gamma(t)+\frac{|\beta(t)|}{\ell(t)}\left(-\frac{\beta(t)}{|\beta(t)|}\right) \nu(t)=\gamma(t)-\frac{\beta(t)}{\ell(t)} \nu(t) .
$$

Second suppose that $t_{0}$ is a singular point of $\gamma$ and $\gamma_{\lambda}$ is a regular curve with $\lambda \neq 1 / \kappa(t)$. Since $\dot{\gamma}_{\lambda}(t)=(\beta(t)+\lambda \ell(t)) \boldsymbol{\mu}(t)$, we have $|\beta(t)+\lambda \ell(t)| \neq 0$ and

$$
\boldsymbol{t}_{\lambda}=\frac{\beta(t)+\lambda \ell(t)}{|\beta(t)+\lambda \ell(t)|} \boldsymbol{\mu}(t), \quad \boldsymbol{n}_{\lambda}=-\frac{\beta(t)+\lambda \ell(t)}{|\beta(t)+\lambda \ell(t)|} \nu(t) .
$$

By Lemma $3.1(2), \kappa_{\lambda}(t)=\ell(t) /|\beta(t)+\lambda \ell(t)|$ and $\ell(t) \neq 0$. Then

$$
\begin{aligned}
\mathcal{E} v\left(\gamma_{\lambda}\right)(t) & =\gamma_{\lambda}(t)+\frac{1}{\kappa_{\lambda}(t)} \boldsymbol{n}_{\lambda}(t)=\gamma(t)+\lambda \nu(t)+\frac{|\beta(t)+\lambda \ell(t)|}{\ell(t)}\left(-\frac{\beta(t)+\lambda \ell(t)}{|\beta(t)+\lambda \ell(t)|}\right) \nu(t) \\
& =\gamma(t)-\frac{\beta(t)}{\ell(t)} \nu(t) .
\end{aligned}
$$


If we take $\widetilde{\nu}(t)=J(\nu(t))=\boldsymbol{\mu}(t)$, then $(\mathcal{E} v(\gamma)(t), \widetilde{\nu}(t))$ is a Legendre immersion. In fact, $\dot{\widetilde{\nu}}(t)=-\ell(t) \nu(t) \neq 0$ and by the form of

$$
\dot{\mathcal{E} v}(\gamma)(t)=-\frac{\dot{\beta}(t) \ell(t)-\beta(t) \dot{\ell}(t)}{\ell(t)^{2}} \nu(t),
$$

we have $\dot{\mathcal{E}} v(\gamma)(t) \cdot \widetilde{\nu}(t)=0$. It follows that $\mathcal{E} v(\gamma)$ is a front. This completes the proof of the Theorem.

Remark 3.4 By the representation (3), we may define the evolute of a front even if $\gamma$ have non-isolated singularities, under the condition $\ell(t) \neq 0$.

By Lemma 3.1 and Remark 3.4, for a Legendre immersion $(\gamma, \nu)$ with the curvature of the Legendre immersion $(\ell, \beta)$, we say that $t_{0}$ is an inflection point of the front $\gamma$ (or, the Legendre immersion $(\gamma, \nu)$ ) if $\ell\left(t_{0}\right)=0$. Since $\beta\left(t_{0}\right) \neq 0$ and Proposition 3.1, $\ell\left(t_{0}\right)=0$ is equivalent to the condition $\kappa\left(t_{0}\right)=0$.

Remark 3.5 Let $(\gamma, \nu)$ be a Legendre immersion, then $(\gamma,-\nu)$ is also (Remark 2.5). However, $\mathcal{E} v(t)$ does not change. It follows that we can define an evolute of a non co-orientable front, by taking double covering of $\gamma$.

Remark 3.6 By Definition 2.9, the evolute of a front is independent on the parametrisation of $(\gamma, \nu)$. The curvature of the Legendre immersion $(\ell, \beta)$ is depended on the parametrisation of $(\gamma, \nu)$, see [7]. If $s=s(t)$ is a parameter changing on $I$ to $\bar{I}$, then $\ell(t)=\ell(s(t)) \dot{s}(t)$ and $\beta(t)=\beta(s(t)) \dot{s}(t)$. It also follows from the representation (3) that the evolute of a front is independent on the parametrisation of $(\gamma, \nu)$.

If $t_{0}$ is a singular point of $\gamma$, then $\beta\left(t_{0}\right)=0$. As a corollary of Theorem 3.3, we have following.

Corollary 3.7 If $t_{0}$ is a singular point of $\gamma$, then $\mathcal{E} v(\gamma)\left(t_{0}\right)=\gamma\left(t_{0}\right)$.

Proposition 3.8 Suppose that $t_{0}$ is a singular point of $\gamma$.

(1) $t_{0}$ is a regular point of $\mathcal{E} v(\gamma)(t)$ if and only if $\gamma$ is diffeomorphic to the $3 / 2$ cusp at $t_{0}$.

(2) $t_{0}$ is a singular point of $\mathcal{E} v(\gamma)(t)$ if and only if $\ddot{\gamma}\left(t_{0}\right)=0$.

Proof. (1) Let $t_{0}$ be a regular point of $\mathcal{E} v(\gamma)(t)$. Since $\beta\left(t_{0}\right)=0$ and $\ell\left(t_{0}\right) \neq 0, \dot{\beta}\left(t_{0}\right) \neq 0$. By the differentiate of $\dot{\gamma}(t)=\beta(t) \boldsymbol{\mu}(t)$, we have

$$
\ddot{\gamma}(t)=\dot{\beta}(t) \boldsymbol{\mu}(t)-\beta(t) \ell(t) \nu(t), \dddot{\gamma}(t)=\left(\ddot{\beta}(t)-\beta(t) \ell(t)^{2}\right) \boldsymbol{\mu}(t)-(2 \dot{\beta}(t) \ell(t)+\beta(t) \dot{\ell}(t)) \nu(t) .
$$

It follows that $\dot{\gamma}\left(t_{0}\right)=0, \ddot{\gamma}\left(t_{0}\right)=\dot{\beta}\left(t_{0}\right) \boldsymbol{\mu}\left(t_{0}\right), \dddot{\gamma}\left(t_{0}\right)=\ddot{\beta}\left(t_{0}\right) \boldsymbol{\mu}\left(t_{0}\right)-2 \dot{\beta}\left(t_{0}\right) \ell\left(t_{0}\right) \nu\left(t_{0}\right)$ and hence

$$
\operatorname{det}\left(\ddot{\gamma}\left(t_{0}\right), \dddot{\gamma}\left(t_{0}\right)\right)=2 \dot{\beta}\left(t_{0}\right)^{2} \ell\left(t_{0}\right) \neq 0 \text {. }
$$

This condition follows that $\gamma$ is diffeomorphic to the $3 / 2$ cusp at $t_{0}$. The converse is also holded by the reversing arguments.

(2) By the proof of $(1), \dot{\beta}\left(t_{0}\right)=0$ if and only if $\ddot{\gamma}\left(t_{0}\right)=0$.

By Proposition 3.8, we can recognize the $3 / 2$ cusp of original curve by the regularity of the evolute of a front, see Examples 6.2 and 6.3.

The most degenerate case of the evolute of a front, we have classified as follows: 
Proposition 3.9 If $\dot{\mathcal{E} v}(\gamma)(t) \equiv 0$, then $\gamma$ is a part of a circle or a point.

Proof. By the condition $\dot{\mathcal{E} v}(\gamma)(t) \equiv 0$, there exists a constant $c \in \mathbb{R}$ such that $\beta(t) / \ell(t) \equiv c$, if and only if $\beta(t)=c \ell(t)$. If $c=0$, then $\dot{\gamma}(t)=\beta(t) \boldsymbol{\mu}(t)=0$. It follows that $\gamma$ is a point. Suppose that $c \neq 0$. By the uniqueness and existence of a front in [7], we take

$$
\nu(t)=\left(\cos \left(\int \ell(t) d t\right), \sin \left(\int \ell(t) d t\right)\right), \boldsymbol{\mu}(t)=\left(-\sin \left(\int \ell(t) d t\right), \cos \left(\int \ell(t) d t\right)\right) .
$$

By $\dot{\gamma}(t)=\beta(t) \boldsymbol{\mu}(t)$, we have

$$
\begin{aligned}
\gamma(t) & =\left(-c \int \ell(t) \sin \left(\int \ell(t) d t\right) d t+a, c \int \ell(t) \cos \left(\int \ell(t) d t\right) d t+b\right) \\
& =\left(c \cos \left(\int \ell(t) d t\right)+a, c \sin \left(\int \ell(t) d t\right)+b\right)
\end{aligned}
$$

for some constants $a, b \in \mathbb{R}$. Therefore $\gamma$ is a part of a circle.

As a well-known result, a singular point of $E v(\gamma)$ of a regular plane curve $\gamma$ is corresponding to a vertex of $\gamma$, namely $\dot{\kappa}(t)=0$ (cf. $[5,8,18,19]$ ).

We extend the notion of vertex. For a Legendre immersion $(\gamma, \nu)$ with the curvature of the Legendre immersion $(\ell, \beta), t_{0}$ is a vertex of the front $\gamma$ (or a Legendre immersion $(\gamma, \nu)$ ) if $(d / d t)(\beta / \ell)\left(t_{0}\right)=0$, namely, $(d / d t) \mathcal{E} v\left(t_{0}\right)=0$. Note that if $t_{0}$ is a regular point of $\gamma$, the definition of the vertex coincides with usual vertex for regular curves. Therefore, this is a generalisation of the notion of the vertex of a regular plane curve.

Remark 3.10 Let $(\gamma, \nu)$ be a Legendre immersion. If $t_{0}$ is a singular point of $\gamma$ which degenerate more than $3 / 2$ cusp, then $t_{0}$ is a vertex of a front $\gamma$. In fact,

$$
\frac{d}{d t}\left(\frac{\beta}{\ell}\right)\left(t_{0}\right)=\frac{\dot{\beta}\left(t_{0}\right) \ell\left(t_{0}\right)-\beta\left(t_{0}\right) \dot{\ell}\left(t_{0}\right)}{\ell\left(t_{0}\right)^{2}}=0
$$

since $\beta\left(t_{0}\right)=\dot{\beta}\left(t_{0}\right)=0$ by Proposition 3.8.

Proposition 3.11 Let $(\gamma, \nu):[0,2 \pi] \rightarrow \mathbb{R}^{2} \times S^{1}$ be a (co-orientable) closed Legendre immersion without inflection points.

(1) If $\gamma$ has at least two singular points which degenerate more than $3 / 2$ cusp, then $\gamma$ has at least four vertices.

(2) If $\gamma$ has at least four singular points, then $\gamma$ has at least four vertices.

Proof. (1) Let $t_{i}$ be a singular point of $\gamma$ for each $i \in\{1, \ldots, n\}$. Suppose that at least two of them are degenerate more than 3/2 cusp. By Remark 3.10, these singularities are vertices of $\gamma$, therefore it is sufficient to show that there is at least one vertex between two adjacent singular points. Since $\gamma$ has no inflection points, the sign of the curvature of $\gamma$ on regular points is constant. Therefore, either $\lim _{t \rightarrow t_{i}} \kappa(t)=\infty$ for all $i \in\{1, \ldots, n\}$ or $\lim _{t \rightarrow t_{i}} \kappa(t)=-\infty$ for all $i \in\{1, \ldots, n\}$. This concludes there exist $t \in\left(t_{i}, t_{i+1}\right)$ such that $\dot{\kappa}(t)=0$ for all $i \in\{1, \ldots, n-1\}$. Moreover, since $\gamma$ is closed, there exists a point $t \in\left[0, t_{1}\right) \cup\left(t_{n}, 2 \pi\right)$ such that $\dot{\kappa}(t)=0$. Therefore, $\gamma$ has at least four vertices.

(2) We can also prove by the same method of (1). 
Finally, in this section, we consider the evolute of a front as a (wave) front of a Legendre immersion by using a family of functions.

We define a family of functions

$$
F: I \times \mathbb{R}^{2} \rightarrow \mathbb{R}
$$

by $F(t, x, y)=(\gamma(t)-(x, y)) \cdot \boldsymbol{\mu}(t)$.

Proposition 3.12 Let $(\gamma, \nu): I \rightarrow \mathbb{R}^{2} \times S^{1}$ be a Legendre immersion with the curvature of the Legendre immersion $(\ell, \beta)$. Then

(1) $F(t, x, y)=0$ if and only if there exists real number $\lambda$ such that $(x, y)=\gamma(t)-\lambda \nu(t)$.

(2) $F(t, x, y)=(\partial F / \partial t)(t, x, y)=0$ if and only if $\ell(t) \neq 0$ and $(x, y)=\gamma(t)-(\beta(t) / \ell(t)) \nu(t)$.

Proof. (1) $(\gamma(t)-(x, y)) \cdot \boldsymbol{\mu}(t)=0$ if and only if there exists $\lambda \in \mathbb{R}$ such that $\gamma(t)-(x, y)=\lambda \nu(t)$.

(2) $(\partial F / \partial t)(t, x, y)=\dot{\gamma}(t) \cdot \boldsymbol{\mu}(t)+(\gamma(t)-(x, y)) \cdot \dot{\boldsymbol{\mu}}(t)=\beta(t)-\lambda \ell(t)$. If $\ell(t)=0$, then $\beta(t)=0$. This is a contradiction for $(\ell(t), \beta(t)) \neq(0,0)$. If follows that $\lambda=\beta(t) / \ell(t)$. The converse is also holded.

One can show that $F$ is a Morse family, in the sense of Legendrian (cf. [1, 14, 20]), namely, $(F, \partial F / \partial t): I \times \mathbb{R}^{2} \rightarrow \mathbb{R} \times \mathbb{R}$ is a submersion at $(t, x, y) \in \Sigma(F)$, where

$$
\Sigma(F)=\{(t, x, y) \mid F(t, x, y)=(\partial F / \partial t)(t, x, y)=0\}
$$

It also follows that the evolute of a front $\mathcal{E} v(\gamma)$ is a (wave) front of a Legendre immersion and is given by envelope of normal lines of the front.

\section{Evolutes of the evolutes of fronts}

By Theorem 3.3, the evolute of a front is also a front. We consider a repeated evolute of an evolute of a front and give properties of a singular point of it.

Theorem 4.1 Let $(\gamma, \nu)$ be a Legendre immersion with the curvature of the Legendre immersion $(\ell, \beta)$. The evolute of an evolute of a front is given by

$$
\mathcal{E} v(\mathcal{E} v(\gamma))(t)=\mathcal{E} v(\gamma)(t)-\frac{\dot{\beta}(t) \ell(t)-\beta(t) \dot{\ell}(t)}{\ell(t)^{3}} \boldsymbol{\mu}(t)
$$

Proof. At this proof, we denote $\widetilde{\gamma}(t)=\mathcal{E} v(\gamma)(t)$. By the proof of Theorem 3.3, $(\widetilde{\gamma}(t), \widetilde{\nu}(t))=$ $(\mathcal{E} v(\gamma)(t), \boldsymbol{\mu}(t))$ is a Legendre immersion. Since $\widetilde{\boldsymbol{\mu}}(t)=J(\widetilde{\nu}(t))=-\nu(t)$ and the derivative of the evolute of the front (4), we have

$$
\widetilde{\beta}(t)=\frac{\dot{\beta}(t) \ell(t)-\beta(t) \dot{\ell}(t)}{\ell(t)^{2}}
$$

where $\dot{\widetilde{\gamma}}(t)=\widetilde{\beta}(t) \widetilde{\boldsymbol{\mu}}(t)$. Moreover $\widetilde{\ell}(t)=\ell(t)$ by the Frenet formula of a front (1). It follows that

$$
\mathcal{E} v(\mathcal{E} v(\gamma))(t)=\mathcal{E} v(\widetilde{\gamma})(t)=\widetilde{\gamma}(t)-\frac{\widetilde{\beta}(t)}{\widetilde{\ell}(t)} \widetilde{\nu}(t)=\mathcal{E} v(\gamma)(t)-\frac{\dot{\beta}(t) \ell(t)-\beta(t) \dot{\ell}(t)}{\ell(t)^{3}} \boldsymbol{\mu}(t)
$$


We can also prove above theorem by a direct calculation of the definition of the evolute of a front (Definition 2.9). We need to divide into four cases, that is, $\gamma$ is a regular or a singular, and $\mathcal{E} v(\gamma)$ is a regular or a singular. All cases coincide with $(5)$. We also call $\mathcal{E} v(\mathcal{E} v(\gamma))$ the second evolute of a front.

Now consider a geometric meaning of a singular point of $\mathcal{E} v(\mathcal{E} v(\gamma))(t)$.

Lemma 4.2 Suppose that $\gamma$ and $\mathcal{E} v(\gamma)$ are regular curves. If $\dot{\mathcal{E} v}(\mathcal{E} v(\gamma))(t) \equiv 0$, then $\gamma$ is an involute of a circle.

Proof. We may assume that $t$ is the arc-length parameter of $\gamma$. It follows that $|\beta(t)|=1$ and hence $\ell(t)=\kappa(t)$ by Lemma 3.1. Moreover, we have $\beta(t)^{2}=1$ and $\dot{\beta}(t)=0$. Since $\boldsymbol{t}(t)=\beta(t) \boldsymbol{\mu}(t), \boldsymbol{n}(t)=-\beta(t) \nu(t)$, we have $\boldsymbol{\mu}(t)=\beta(t) \boldsymbol{t}(t)$ and $\nu(t)=-\beta(t) \boldsymbol{n}(t)$. Then

$$
\mathcal{E} v(\gamma)(t)=\gamma(t)-\frac{\beta(t)}{\ell(t)} \nu(t)=\gamma(t)-\frac{\beta(t)}{\kappa(t)}(-\beta(t) \boldsymbol{n}(t))=\gamma(t)+\frac{1}{\kappa(t)} \boldsymbol{n}(t)
$$

and

$$
\mathcal{E} v(\mathcal{E} v(\gamma))(t)=\mathcal{E} v(\gamma)(t)+\frac{\beta(t) \dot{\kappa}(t)}{\kappa(t)^{3}} \beta(t) \boldsymbol{t}(t)=\mathcal{E} v(\gamma)(t)+\frac{\dot{\kappa}(t)}{\kappa(t)^{3}} \boldsymbol{t}(t)
$$

It follows that

$$
\dot{\mathcal{E} v}(\gamma)(t)=-\frac{\dot{\kappa}(t)}{\kappa(t)^{2}} \boldsymbol{n}(t), \dot{\mathcal{E} v}(\mathcal{E} v(\gamma))(t)=\frac{\ddot{\kappa}(t) \kappa(t)-3 \dot{\kappa}(t)^{2}}{\kappa(t)^{4}} \boldsymbol{t}(t)
$$

By the assumptions, $\kappa(t) \neq 0, \dot{\kappa}(t) \neq 0$ and $\ddot{\kappa}(t) \kappa(t)-3 \dot{\kappa}(t)^{2} \equiv 0$. It follows that

$$
\frac{d}{d t}\left(\frac{\dot{\kappa}(t)}{\kappa(t)}\right)=2\left(\frac{\dot{\kappa}(t)}{\kappa(t)}\right)^{2}
$$

Solving the differential equation, there exist constants $C_{1}, C_{2} \in \mathbb{R}$ with $C_{2} \neq 0$ such that

$$
\kappa(t)=C_{2} \frac{1}{\sqrt{2 t+C_{1}}} .
$$

A curve with the curvature $1 / \sqrt{2 c t}$ for a constant $c \in \mathbb{R}$ is an involute of circle with radius $c$. By the existence and uniqueness theorem of regular plane curves, see for example [8,9], $\gamma$ is an involute of a circle (cf. [9, P.138]).

Let $\gamma: I \rightarrow \mathbb{R}^{2}$ be a regular curve and $t_{0} \in I$. The involute of a regular curve is defined by $\operatorname{Inv}\left(\gamma, t_{0}\right): I \rightarrow \mathbb{R}^{2}$

$$
\operatorname{Inv}\left(\gamma, t_{0}\right)(t)=\gamma(t)-\left(\int_{t_{0}}^{t}|\dot{\gamma}(t)| d t\right) \boldsymbol{t}(t) .
$$

Note that $\mathcal{E} v\left(\operatorname{Inv}\left(\gamma, t_{0}\right)\right)(t)=\gamma(t)$, for more detail see $[5,8,9]$.

Theorem 4.3 Suppose that $\gamma$ and $\mathcal{E} v(\gamma)$ are regular curves. If $t_{0}$ is a singular point of $\mathcal{E} v(\mathcal{E} v(\gamma))$, then $\gamma$ is at least 4-th order contact to an involute of a circle at the point $t=t_{0}$ up to congruent. 
Proof. We may assume that $t$ is the arc-length parameter of $\gamma$. By the same arguments in the proof of Lemma 4.2 , we have $\kappa\left(t_{0}\right) \neq 0, \dot{\kappa}\left(t_{0}\right) \neq 0$ and $\ddot{\kappa}\left(t_{0}\right) \kappa\left(t_{0}\right)-3 \dot{\kappa}\left(t_{0}\right)^{2}=0$. We put on $\kappa\left(t_{0}\right)=a$ and $\dot{\kappa}\left(t_{0}\right)=b$. Then we define a curve $\widetilde{\gamma}(t)$ whose curvature is given by

$$
\widetilde{\kappa}(t)=a \sqrt{\frac{a}{b}} \frac{1}{\sqrt{-2 t+2 t_{0}+\frac{a}{b}}}, \quad\left(\text { respectively, } \widetilde{\kappa}(t)=a \sqrt{-\frac{a}{b}} \frac{1}{\sqrt{2 t-2 t_{0}-\frac{a}{b}}}\right)
$$

if $a b>0$ (respectively, $a b<0$ ). Then $\kappa\left(t_{0}\right)=\widetilde{\kappa}\left(t_{0}\right)=a$ and $\dot{\kappa}\left(t_{0}\right)=\dot{\tilde{\kappa}}\left(t_{0}\right)=b$. Since $\ddot{\kappa}\left(t_{0}\right) \kappa\left(t_{0}\right)-3 \dot{\kappa}\left(t_{0}\right)^{2}=0$ and $\ddot{\widetilde{\kappa}}(t) \widetilde{\kappa}(t)-3 \dot{\widetilde{\kappa}}(t)^{2} \equiv 0$, we have $\ddot{\kappa}\left(t_{0}\right)=\ddot{\widetilde{\kappa}}\left(t_{0}\right)$. By the Theorem A.1 in the appendix, $\gamma$ and $\widetilde{\gamma}$ are at least 4 -th order contact at the point $t=t_{0}$ up to congruent. It follows that $\gamma$ and an involute of a circle are at least 4 -th order contact at the point $t=t_{0}$ up to congruent.

This completes the proof of Theorem.

Remark 4.4 Suppose that $\gamma$ is a regular curve. If $t_{0}$ is a singular point of $\mathcal{E} v(\gamma)(t)$ and $\mathcal{E} v(\mathcal{E} v(\gamma))(t)$, then $\dot{\kappa}\left(t_{0}\right)=\ddot{\kappa}\left(t_{0}\right)=0$ by the same calculations of the proof of Lemma 4.2 . It follows that $\gamma$ and the osculating circle are at least 4 -th order contact at the point $t=t_{0}$.

Proposition 4.5 Suppose that $t_{0}$ is a singular point of both $\gamma$ and $\mathcal{E} v(\gamma)$.

(1) $t_{0}$ is a regular point of $\mathcal{E} v(\mathcal{E} v(\gamma))$ if and only if $\gamma$ is diffeomorphic to the $4 / 3$ cusp at $t_{0}$.

(2) $t_{0}$ is a singular point of $\mathcal{E} v(\mathcal{E} v(\gamma))$ if and only if $\dddot{\gamma}\left(t_{0}\right)=0$.

Proof. (1) Let $t_{0}$ be a regular point of $\mathcal{E} v(\mathcal{E} v(\gamma))$. By Proposition 3.8, $\beta\left(t_{0}\right)=\dot{\beta}\left(t_{0}\right)=0$ and $\ell\left(t_{0}\right) \neq 0$. Then $\dot{\gamma}\left(t_{0}\right)=\ddot{\gamma}\left(t_{0}\right)=0$. Since

$$
\frac{d}{d t} \mathcal{E} v(\mathcal{E} v(\gamma))(t)=-\frac{\ddot{\beta}(t) \ell(t)^{2}-\beta(t) \ell(t) \ddot{\ell}(t)-3 \dot{\beta}(t) \ell(t) \dot{\ell}(t)+3 \beta(t) \dot{\ell}(t)^{2}}{\ell(t)^{4}},
$$

$(d / d t) \mathcal{E} v(\mathcal{E} v(\gamma))\left(t_{0}\right)=-\ddot{\beta}\left(t_{0}\right) \ell\left(t_{0}\right)^{-2} \neq 0$, if and only if $\ddot{\beta}\left(t_{0}\right) \neq 0$. By the differentiate of $\dot{\gamma}(t)=\beta(t) \boldsymbol{\mu}(t)$, we have

$$
\begin{aligned}
\dddot{\gamma}(t)= & \left(\ddot{\beta}(t)-\beta(t) \ell(t)^{2}\right) \boldsymbol{\mu}(t)-(2 \dot{\beta}(t) \ell(t)+\beta(t) \dot{\ell}(t)) \nu(t), \\
\gamma^{(4)}(t)= & \left(\dddot{\beta}(t)-3 \dot{\beta}(t) \ell(t)^{2}-3 \beta(t) \ell(t) \dot{\ell}(t)\right) \boldsymbol{\mu}(t) \\
& \quad-\left(3 \ddot{\beta}(t) \ell(t)-\beta(t) \ell(t)^{3}+3 \dot{\beta}(t) \dot{\ell}(t)+\beta(t) \ddot{\ell}(t)\right) \nu(t) .
\end{aligned}
$$

It follows that $\dddot{\gamma}\left(t_{0}\right)=\ddot{\beta}\left(t_{0}\right) \boldsymbol{\mu}\left(t_{0}\right), \gamma^{(4)}\left(t_{0}\right)=\dddot{\beta}\left(t_{0}\right) \boldsymbol{\mu}\left(t_{0}\right)-3 \ddot{\beta}\left(t_{0}\right) \ell\left(t_{0}\right) \nu\left(t_{0}\right)$ and hence

$$
\operatorname{det}\left(\dddot{\gamma}\left(t_{0}\right), \gamma^{(4)}\left(t_{0}\right)\right)=3 \ddot{\beta}\left(t_{0}\right)^{2} \ell\left(t_{0}\right) \neq 0 \text {. }
$$

This condition follows that $\gamma$ is diffeomorphic to the $4 / 3$ cusp at $t_{0}$ (cf. [4, 11, 12]). The converse is also holded by the reversing arguments.

(2) By the proof of (1), $\ddot{\beta}\left(t_{0}\right)=0$ if and only if $\dddot{\gamma}\left(t_{0}\right)=0$.

\section{The $n$-th evolutes of fronts}

We give the form of the $n$-th evolute of a front, where $n$ is a natural number. We denote $\mathcal{E} v^{0}(\gamma)(t)=\gamma(t)$ for convenience, $\mathcal{E} v^{1}(\gamma)(t)=\mathcal{E} v(\gamma)(t)$ and $\mathcal{E} v^{n}(\gamma)(t)=\mathcal{E} v\left(\mathcal{E} v^{n-1}(\gamma)\right)(t)$ inductively. We define $\beta_{0}(t)=\beta(t) / \ell(t)$ and $\beta_{n}(t)=\dot{\beta}_{n-1}(t) / \ell(t)$ inductively. 
Theorem 5.1 Let $(\gamma, \nu): I \rightarrow \mathbb{R}^{2} \times S^{1}$ be a Legendre immersion with the curvature of the Legendre immersion $(\ell, \beta)$. The $n$-th evolute of a front is given by

$$
\mathcal{E} v^{n}(\gamma)(t)=\mathcal{E} v^{n-1}(\gamma)(t)-\beta_{n-1}(t) J^{n-1}(\nu(t)),
$$

where $J^{n-1}$ is $(n-1)$-times of $J$.

Proof. Let $n=1$ and $n=2$, then

$$
\mathcal{E} v^{1}(\gamma)(t)=\mathcal{E} v^{0}(\gamma)(t)-\beta_{0}(t) J^{0}(\nu(t))=\gamma(t)-\frac{\beta(t)}{\ell(t)} \nu(t)
$$

and

$$
\begin{aligned}
\mathcal{E} v^{2}(\gamma)(t) & =\mathcal{E} v^{1}(\gamma)(t)-\beta_{1}(t) J^{1}(\nu(t))=\mathcal{E} v(\gamma)(t)-\frac{d}{d t}\left(\frac{\beta(t)}{\ell(t)}\right) \frac{1}{\ell(t)} J(\nu(t)) \\
& =\mathcal{E} v(\gamma)(t)-\frac{\dot{\beta}(t) \ell(t)-\beta(t) \dot{\ell}(t)}{\ell(t)^{3}} \boldsymbol{\mu}(t) .
\end{aligned}
$$

These are nothing but the evolute of a front (3) and the second evolute of a front (5).

Next suppose that $1 \leq j \leq k$ is holded, namely,

$$
\mathcal{E} v^{j}(\gamma)(t)=\mathcal{E} v^{j-1}(\gamma)(t)-\beta_{j-1}(t) J^{j-1}(\nu(t))
$$

for $1 \leq j \leq k$. We consider $\mathcal{E} v\left(\mathcal{E} v^{k}(\gamma)\right)(t)$. Since $\left(\mathcal{E} v^{k}(\gamma)(t), J^{k}(\nu(t))\right)$ is a Legendre immersion,

$$
\begin{aligned}
\frac{d}{d t} \mathcal{E} v^{k}(\gamma)(t) & =-\dot{\beta}_{k-2}(t) J^{k-2}(\nu(t))-\dot{\beta}_{k-1}(t) J^{k-1}(\nu(t))-\beta_{k-1}(t) J^{k-1}(\dot{\nu}(t)) \\
& =-\dot{\beta}_{k-2}(t) J^{k-2}(\nu(t))-\dot{\beta}_{k-1}(t) J^{k-1}(\nu(t))-\dot{\beta}_{k-2}(t) J^{k}(\nu(t)) \\
& =-\dot{\beta}_{k-1}(t) J^{k-1}(\nu(t)) \\
& =\dot{\beta}_{k-1}(t) J^{k+1}(\nu(t)) .
\end{aligned}
$$

Then, by Theorem 3.3, we have $(k+1)$-th evolute of the front

$$
\mathcal{E} v^{k+1}(\gamma)(t)=\mathcal{E} v^{k}(\gamma)(t)-\frac{\dot{\beta}_{k-1}(t)}{\ell(t)} J^{k}(\nu(t))=\mathcal{E} v^{k}(\gamma)(t)-\beta_{k}(t) J^{k}(\nu(t))
$$

By the induction, this completes the proof of Theorem.

As a generalisation of Propositions 3.8 (2) and 4.5 (2), we have the following result:

Proposition 5.2 Let $(\gamma, \nu): I \rightarrow \mathbb{R}^{2} \times S^{1}$ be a Legendre immersion with the curvature of the Legendre immersion $(\ell, \beta)$. Suppose that $t_{0}$ is a singular point of $\gamma$. Then the following are equivalent:

(1) $t_{0}$ is a singular point of $\mathcal{E} v^{i}(\gamma)(t)$ for $i=1, \ldots, n$.

(2) $\left(d^{i} \beta / d t^{i}\right)\left(t_{0}\right)=0$ for $i=1, \ldots, n$.

(3) $\left(d^{i} \gamma / d t^{i}\right)\left(t_{0}\right)=0$ for $i=2, \ldots, n+1$. 
Proof. First, we show that $\beta_{i}(t)$ is given by the form $\beta^{(i)}(t)$ and lower terms of $\beta^{(i)}(t)$, namely,

$$
\beta_{i}(t)=\frac{\beta^{(i)}(t)}{\ell(t)^{i+1}}+L\left(\beta(t), \ldots, \beta^{(i-1)}(t)\right)
$$

for some smooth function $L$ which contain $\ell(t)$ and derivatives of $\ell(t)$.

Since $\beta_{1}(t)=\dot{\beta}_{0}(t) / \ell(t)=\beta^{(1)}(t) / \ell(t)^{2}+(\beta(t) / \ell(t))(d / d t)(1 / \ell(t))$, the case of $i=1$ is holded. Suppose that $i=k$ is holded, namely, there exists a smooth function $L$ such that

$$
\beta_{k}(t)=\frac{\beta^{(k)}(t)}{\ell(t)^{k+1}}+L\left(\beta(t), \ldots, \beta^{(k-1)}(t)\right) .
$$

Then

$$
\beta_{k+1}(t)=\frac{\dot{\beta}_{k}(t)}{\ell(t)}=\frac{\beta^{(k+1)}(t)}{\ell(t)^{k+2}}+\widetilde{L}\left(\beta(t), \ldots, \beta^{(k)}(t)\right),
$$

for some smooth function $\widetilde{L}$. By the induction, we conclude the assertion.

Second, assume that $t_{0}$ is a singular point of $\mathcal{E} v^{i}(\gamma)(t)$ for $i=1, \ldots, n$. By Theorem 5.1, $(d / d t) \mathcal{E} v^{i}(\gamma)\left(t_{0}\right)=0$ if and only if $\beta_{i}\left(t_{0}\right)=0$. Since $(6)$ and $\beta\left(t_{0}\right)=0, \beta_{i}\left(t_{0}\right)=0$ for $i=1, \ldots, n$ if and only if $\beta^{(i)}\left(t_{0}\right)=0$ for $i=1, \ldots, n$. It follows that (1) implies (2). By the reversing arguments, the converse (1) follows from (2).

Finally, since $\dot{\gamma}(t)=\beta(t) \boldsymbol{\mu}(t)$, we can show that (2) is equivalent to (3) by using the induction.

\section{$6 \quad$ Examples}

We give examples to understand the phenomena for evolutes of fronts.

Example 6.1 Let $\gamma(t)=(a \cos t, b \sin t), a \neq b$ be an ellipse. Since

$$
\nu(t)=\frac{1}{\sqrt{a^{2} \sin ^{2} t+b^{2} \cos ^{2} t}}(-b \cos t, a \sin t), \boldsymbol{\mu}(t)=\frac{1}{\sqrt{a^{2} \sin ^{2} t+b^{2} \cos ^{2} t}}(-a \sin t,-b \cos t),
$$

we have

$$
\ell(t)=\frac{a b}{a^{2} \sin ^{2} t+b^{2} \cos ^{2} t}, \beta(t)=-\sqrt{a^{2} \sin ^{2} t+b^{2} \cos ^{2} t} .
$$

The evolute, the second evolute and the third evolute of the ellipse are given by

$$
\begin{aligned}
\mathcal{E} v(\gamma)(t)= & \left(\frac{a^{2}-b^{2}}{a} \cos ^{3} t,-\frac{a^{2}-b^{2}}{b} \sin ^{3} t\right), \\
\mathcal{E} v(\mathcal{E} v(\gamma))(t)= & \left(\frac{a^{2}-b^{2}}{a b^{2}} \cos t\left(b^{2} \cos ^{4} t+3 a^{2} \sin ^{4} t+b^{2} \sin ^{2} 2 t\right),\right. \\
& \quad-\frac{a^{2}-b^{2}}{a^{2} b} \sin t\left(a^{2} \sin ^{4} t+3 b^{2} \cos ^{4} t+a^{2} \sin ^{2} 2 t\right),
\end{aligned}
$$

and $\mathcal{E} v^{3}(\gamma)(t)=$

$$
\begin{gathered}
\left(\frac{a^{2}-b^{2}}{8 a^{3} b^{2}} \cos ^{3} t\left(45 a^{4}-10 a^{2} b^{2}-3 b^{4}+12\left(-5 a^{4}+4 a^{2} b^{2}+b^{4}\right) \cos 2 t+15\left(a^{2}-b^{2}\right)^{2} \cos 4 t\right)\right. \\
\left.\frac{a^{2}-b^{2}}{8 a^{2} b^{3}} \sin ^{3} t\left(3 a^{4}+10 a^{2} b^{2}-45 b^{4}+12\left(a^{4}+4 a^{2} b^{2}-5 b^{4}\right) \cos 2 t-15\left(a^{2}-b^{2}\right)^{2} \cos 4 t\right)\right) .
\end{gathered}
$$


The ellipse and its evolute (red curve), see Figures 1 left and 2 centre. Moreover, the second evolute (yellow curve), see Figure 1 centre, and the third evolute (green curve), see Figures 1 right and 2 right.

The evolute is useful to recognize the difference of the sharp of curves. In figure 2, the left is a circle and the centre is an ellipse and its evolute. We can observe the evolute of the ellipse, however, it is very small (red curve). If we consider the repeated evolute, we can easy to observe it. The right in figure 2 is the second and the third evolute of the ellipse.
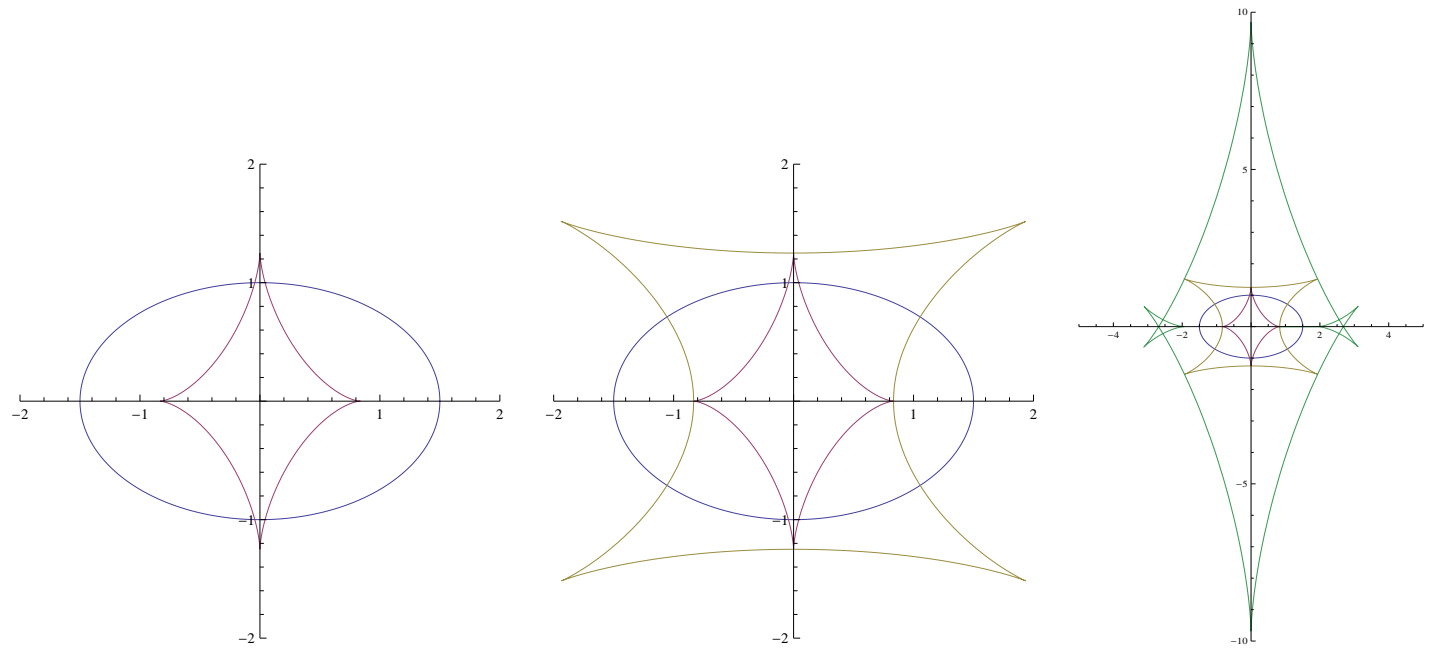

Figure 1. The ellipse and evolutes.
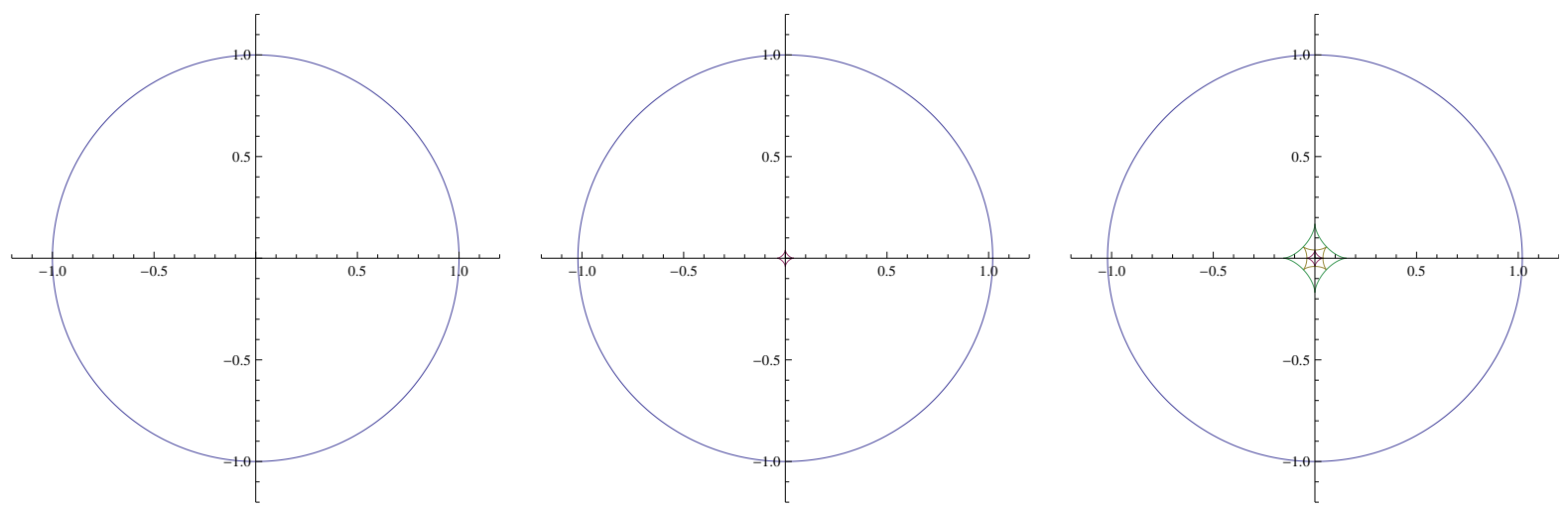

Figure 2.

Example 6.2 Let $\gamma(t)=(3 \cos t-\cos 3 t, 3 \sin t-\sin 3 t)=\left(6 \cos t-4 \cos ^{3} t, 4 \sin ^{3} t\right)$ be the nephroid, see Figure 3 left. Since $\nu(t)=(-\sin 2 t, \cos 2 t), \boldsymbol{\mu}(t)=(-\cos 2 t, \sin 2 t)$, we have $\ell(t)=2, \beta(t)=-6 \sin t$. The evolute and the second evolute of the nephroid are as follows, see Figure 3 centre and right:

$$
\begin{aligned}
\mathcal{E} v(\gamma)(t) & =\left(2 \cos ^{3} t, 3 \sin t-2 \sin ^{2} t\right) \\
\mathcal{E} v(\mathcal{E} v(\gamma))(t) & =\left(\frac{3}{2} \cos t-\cos ^{3} t, \sin ^{3} t\right) .
\end{aligned}
$$

We can observe that $\gamma(t) / 4=\mathcal{E} v(\mathcal{E} v(\gamma))(t)$. 

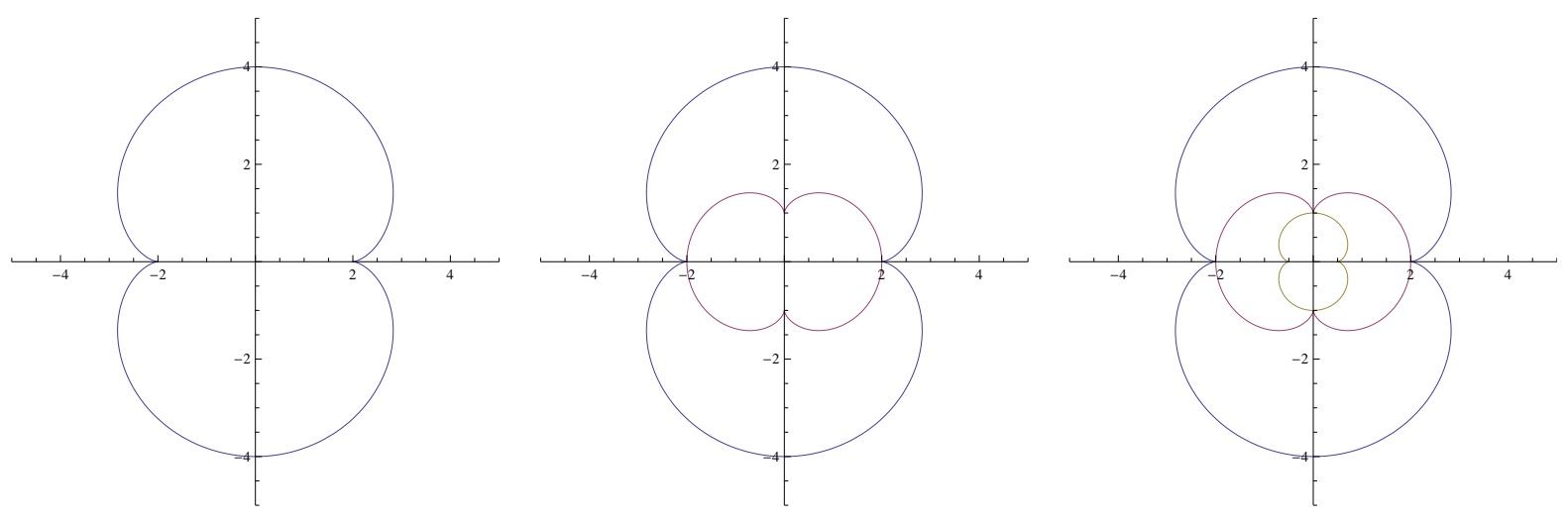

Figure 3. The nephroid and evolutes.

Example 6.3 Let $\gamma(t)=\left(t^{3}, t^{4}\right)$ be the $4 / 3$-cusp, Figure 4 left. Since $\nu(t)=\left(1 / \sqrt{16 t^{2}+9}\right)(-4 t, 3)$, $\boldsymbol{\mu}(t)=\left(1 / \sqrt{16 t^{2}+9}\right)(-3,-4 t)$, we have $\ell(t)=12 /\left(16 t^{2}+9\right), \beta(t)=-t^{2} \sqrt{16 t^{2}+9}$. The evolute and the second evolute of the $4 / 3$-cusp are as follows, see Figure 4 centre and right:

$$
\begin{aligned}
\mathcal{E} v(\gamma)(t) & =\left(-2 t^{3}-\frac{16}{3} t^{5}, \frac{9}{4} t^{2}+5 t^{4}\right) \\
\mathcal{E} v(\mathcal{E} v(\gamma))(t) & =\left(-\frac{27}{8} t-23 t^{3}-32 t^{5},-\frac{9}{4} t^{2}-23 t^{4}-\frac{320}{9} t^{6}\right) .
\end{aligned}
$$
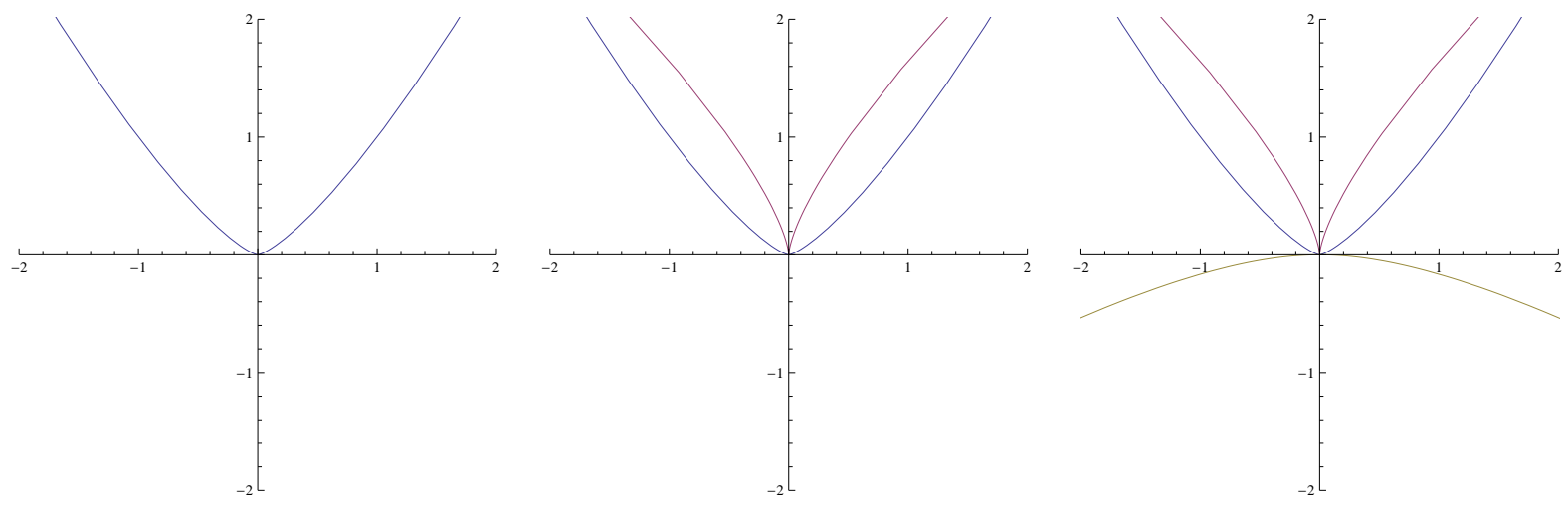

Figure 4. The 4/3-cusp and evolutes.

\section{A Contact between curves}

In this appendix, we discuss contact between regular curves. Let $\gamma: I \rightarrow \mathbb{R}^{2} ; t \mapsto \gamma(t)$ and $\widetilde{\gamma}: \widetilde{I} \rightarrow \mathbb{R}^{2} ; u \mapsto \widetilde{\gamma}(u)$ be regular plane curves, respectively. We say that $\gamma$ and $\widetilde{\gamma}$ are $k$-th order contact at $t=t_{0}, u=u_{0}$ if

$$
\gamma\left(t_{0}\right)=\widetilde{\gamma}\left(u_{0}\right), \frac{d \gamma}{d t}\left(t_{0}\right)=\frac{d \widetilde{\gamma}}{d u}\left(u_{0}\right), \cdots, \frac{d^{k} \gamma}{d t^{k}}\left(t_{0}\right)=\frac{d^{k} \widetilde{\gamma}}{d u^{k}}\left(u_{0}\right), \frac{d^{k+1} \gamma}{d t^{k+1}}\left(t_{0}\right) \neq \frac{d^{k+1} \widetilde{\gamma}}{d u^{k+1}}\left(u_{0}\right)
$$

Moreover, we say that $\gamma$ and $\widetilde{\gamma}$ are at least $k$-th order contact at $t=t_{0}, u=u_{0}$ if

$$
\gamma\left(t_{0}\right)=\widetilde{\gamma}\left(u_{0}\right), \frac{d \gamma}{d t}\left(t_{0}\right)=\frac{d \widetilde{\gamma}}{d u}\left(u_{0}\right), \cdots, \frac{d^{k} \gamma}{d t^{k}}\left(t_{0}\right)=\frac{d^{k} \widetilde{\gamma}}{d u^{k}}\left(u_{0}\right)
$$


Let $\gamma_{1}, \gamma_{2}: I \rightarrow \mathbb{R}^{2}$ be regular plane curves. We say that $\gamma_{1}$ and $\gamma_{2}$ are congruent if there exists a congruence $C$ such that $\gamma_{2}(t)=C\left(\gamma_{1}(t)\right)$ for all $t \in I$. A congruence is given by composition of a rotation and a translation.

Let $\gamma: I \rightarrow \mathbb{R}^{2} ; t \mapsto \gamma(t)$ and $\widetilde{\gamma}: \widetilde{I} \rightarrow \mathbb{R}^{2} ; u \mapsto \widetilde{\gamma}(u)$ be regular plane curves. We take the arc-length parameter for $\gamma(t)$ and $\widetilde{\gamma}(u)$, respectively. In general, we may assume that $\gamma(t)$ and $\widetilde{\gamma}(u)$ are at least first order contact at any point $t=t_{0}, u=u_{0}$ up to congruent. We denote the curvatures $\kappa(t)$ of $\gamma(t)$ and $\widetilde{\kappa}(u)$ of $\widetilde{\gamma}(u)$, respectively.

Theorem A.1 Let $\gamma: I \rightarrow \mathbb{R}^{2}$ and $\widetilde{\gamma}: \widetilde{I} \rightarrow \mathbb{R}^{2}$ be regular plane curves. If $\gamma(t)$ and $\widetilde{\gamma}(u)$ are at least $(k+2)$-th order contact at $t=t_{0}, u=u_{0}$ then

$$
\kappa\left(t_{0}\right)=\widetilde{\kappa}\left(u_{0}\right), \frac{d \kappa}{d t}\left(t_{0}\right)=\frac{d \widetilde{\kappa}}{d u}\left(u_{0}\right), \cdots, \frac{d^{k} \kappa}{d t^{k}}\left(t_{0}\right)=\frac{d^{k} \widetilde{\kappa}}{d u^{k}}\left(u_{0}\right) .
$$

Conversely, if $t$ and $u$ are the arc-length parameter of $\gamma$ and $\widetilde{\gamma}$ respectively, and the condition (7) holds, then $\gamma$ and $\widetilde{\gamma}$ are at least $(k+2)$-th order contact at $t=t_{0}, u=u_{0}$ up to congruent.

Proof. We may assume that $t$ and $u$ are the arc-length parameter of $\gamma$ and $\widetilde{\gamma}$ respectively. Suppose that $\gamma$ and $\widetilde{\gamma}$ are at least third order contact. Since the Frenet formula, we have $d \gamma / d t(t)=\boldsymbol{t}(t), d^{2} \gamma / d t^{2}(t)=\kappa(t) \boldsymbol{n}(t)$ and $d \widetilde{\gamma} / d u(u)=\widetilde{\boldsymbol{t}}(u), d^{2} \widetilde{\gamma} / d u^{2}(u)=\widetilde{\kappa}(u) \widetilde{\boldsymbol{n}}(u)$. It follows that $\boldsymbol{t}\left(t_{0}\right)=\widetilde{\boldsymbol{t}}\left(u_{0}\right), \boldsymbol{n}\left(t_{0}\right)=\widetilde{\boldsymbol{n}}\left(u_{0}\right)$ and $\kappa\left(t_{0}\right)=\widetilde{\kappa}\left(u_{0}\right)$. Hence, the case of $k=1$ holds.

Suppose that $\gamma$ and $\widetilde{\gamma}$ are at least $(k+2)$-th order contact and

$$
\kappa\left(t_{0}\right)=\widetilde{\kappa}\left(u_{0}\right), \frac{d \kappa}{d t}\left(t_{0}\right)=\frac{d \widetilde{\kappa}}{d u}\left(u_{0}\right), \cdots, \frac{d^{k-1} \kappa}{d t^{k-1}}\left(t_{0}\right)=\frac{d^{k-1} \widetilde{\kappa}}{d u^{k-1}}\left(u_{0}\right)
$$

hold. Since $\left(d^{3} \gamma / d t^{3}\right)(t)=(d \kappa / d t)(t) \boldsymbol{n}(t)-\kappa(t)^{2} \boldsymbol{t}(t)$, the form of $\left(d^{k+1} \gamma / d t^{k+1}\right)(t)$ is given by

$$
\frac{d^{k-1} \kappa}{d t^{k-1}}(t) \boldsymbol{n}(t)+f\left(\kappa(t), \cdots, \frac{d^{k-2} \kappa}{d t^{k-2}}(t)\right) \boldsymbol{t}(t)+g\left(\kappa(t), \cdots, \frac{d^{k-2} \kappa}{d t^{k-2}}(t)\right) \boldsymbol{n}(t),
$$

for some smooth functions $f$ and $g$. Then

$$
\frac{d^{k+2} \gamma}{d t^{k+2}}(t)=\frac{d^{k} \kappa}{d t^{k}}(t) \boldsymbol{n}(t)+F\left(\kappa(t), \cdots, \frac{d^{k-1} \kappa}{d t^{k-1}}(t)\right) \boldsymbol{t}(t)+G\left(\kappa(t), \cdots, \frac{d^{k-1} \kappa}{d t^{k-1}}(t)\right) \boldsymbol{n}(t)
$$

for some smooth functions $F$ and $G$. By the same calculations, we have

$$
\frac{d^{k+2} \widetilde{\gamma}}{d u^{k+2}}(u)=\frac{d^{k} \widetilde{\kappa}}{d u^{k}}(u) \widetilde{\boldsymbol{n}}(u)+F\left(\widetilde{\kappa}(u), \cdots, \frac{d^{k-1} \widetilde{\kappa}}{d u^{k-1}}(u)\right) \widetilde{\boldsymbol{t}}(u)+G\left(\widetilde{\kappa}(u), \cdots, \frac{d^{k-1} \widetilde{\kappa}}{d u^{k-1}}(u)\right) \widetilde{\boldsymbol{n}}(u) .
$$

It follows that $\left(d^{k} \kappa / d t^{k}\right)\left(t_{0}\right)=\left(d^{k} \widetilde{\kappa} / d u^{k}\right)\left(u_{0}\right)$. By the induction, we have the first assertion.

By the reversing arguments, we can prove the converse assertion up to congruent.

\section{References}

[1] V. I. Arnol'd, S. M. Gusein-Zade and A. N. Varchenko, Singularities of Differentiable Maps vol. I. Birkhäuser (1986). 
[2] V. I. Arnol'd, Singularities of Caustics and Wave Fronts. Mathematics and Its Applications 62 Kluwer Academic Publishers (1990).

[3] V. I. Arnol'd, Topological properties of Legendre projections in contact geometry of wave fronts.. St. Petersburg Math. J. 6 (1995), 439-452.

[4] J. W. Bruce and T. J. Gaffney, Simple singularities of mappings $\mathbb{C}, 0 \rightarrow \mathbb{C}^{2}, 0$. J. London Math. Soc. (2). 26 (1982), 465-474.

[5] J. W. Bruce and P. J. Giblin, Curves and singularities. A geometrical introduction to singularity theory. Second edition. Cambridge University Press, Cambridge, 1992.

[6] J. Ehlers and E. T. Newman, The theory of caustics and wave front singularities with physical applications, J. Math. Physics. 41 (2000), 3344-3378.

[7] T. Fukunaga and M. Takahashi, Existence and uniqueness for Legendre curves. Preprint. (2012).

[8] C. G. Gibson, Elementary geometry of differentiable curves. An undergraduate introduction. Cambridge University Press, Cambridge, 2001.

[9] A. Gray, E. Abbena and S. Salamon, Modern differential geometry of curves and surfaces with Mathematica. Third edition. Studies in Advanced Mathematics. Chapman and Hall/CRC, Boca Raton, FL, 2006

[10] W. Hasse, M. Kriele and V. Perlick, Caustics of wavefronts in general relativity, Class. Quantum Grav. 13 (1996), 1161-1182.

[11] G. Ishikawa, Zariski's moduli problem for plane branches and the classification of Legendre curve singularities. Real and complex singularities, World Sci. Publ. Hackensack, NJ, (2007), 56-84.

[12] G. Ishikawa and S. Janeczko, Symplectic bifurcations of plane curves and isotropic liftings. $Q$. J. Math. 54 (2003), 73-102.

[13] S. Izumiya, D. Pei, T. Sano and E. Torii, Evolutes of hyperbolic plane curves. Acta Math. Sin. (Engl. Ser.) 20 (2004), 543-550.

[14] S. Izumiya and M. Takahashi, Caustics and wave front propagations: applications to differential geometry. Geometry and topology of caustics-CAUSTICS '06, Banach Center Publ., 82, (2008) $125-142$.

[15] K. Saji, M. Umehara and K. Yamada, The geometry of fronts. Ann. of Math. 169 (2009), 491-529.

[16] K. Saji, M. Umehara and K. Yamada, The duality between singular points and inflection points on wave fronts. Osaka J. Math. 47 (2010), 591-607.

[17] A. Saloom and F. Tari, Curves in the Minkowski plane and their contact with pseudo-circles. Geom. Dedicata. 159 (2012), 109-124.

[18] M. Umehara, A computation of the basic invariant $J^{+}$for closed 2-vertex curves, J. Knot Theory Ramifications 6 (1997), 105-113.

[19] M. Umehara, A unified approach to the four vertex theorems. I. Differential and symplectic topology of knots and curves, Amer. Math. Soc. Transl. Ser. 2, 190, (1999) 185-228. 
[20] V. M. Zakalyukin, Reconstructions of fronts and caustics depending on a parameter and versality of mappings. J. Soviet Math. 27 (1983), 2713-2735.

Tomonori Fukunaga,

Department of Mathematics, Hokkaido University, Sapporo 060-0810, Japan,

E-mail address: fukunaga@math.sci.hokudai.ac.jp

Masatomo Takahashi,

Muroran Institute of Technology, Muroran 050-8585, Japan,

E-mail address: masatomo@mmm.muroran-it.ac.jp 